\title{
Inhibition effects of total flavonoids from Scutellaria barbata D. Don on human breast carcinoma bone metastasis via downregulating PTHrP pathway
}

\author{
XIAO ZHENG ${ }^{*}$, WEN KANG ${ }^{*}$, HUIHUI LIU and SHANYU GUO \\ Department of General Surgery, Shanghai Ninth People's Hospital, \\ Shanghai Jiao Tong University School of Medicine, Shanghai 200011, P.R. China
}

Received October 4, 2016; Accepted January 29, 2018

DOI: $10.3892 /$ ijmm.2018.3515

\begin{abstract}
It is abundantly clear that tumor-derived parathyroid hormone-related protein (PTHrP), receptor activator of nuclear factor- $\kappa \mathrm{B}$ ligand (RANKL) and osteoprotegerin (OPG) are central contributors in promoting osteolytic process of breast carcinoma bone metastasis. Forcusing on this molecular basis, the study was undertaken to explore the inhibition effects of total flavonoids from Scutellaria barbata D. Don (TF-SB) on human breast carcinoma bone metastasis. MDA-MB-231 cells and nude mouse models of breast cancer bone metastasis were given TF-SB in different concentrations. The proliferation, migration and invasion potentials of MDA-MB-231 cells were respectively tested. The effects of TF-SB on tumor weights and bone destruction were investigated. The mRNA and protein expression of PTHrP, OPG and RANKL were assessed by qPCR and western blot analysis. In vitro, TF-SB inhibited the proliferation, migration and invasion of MDA-MB-231 cells in a dose-dependent manner. In vivo, TF-SB prevented bone metastasis of breast cancer by decreasing the number of osteoclast cells per field in a dose-dependent manner, but not affecting tumor growth or mouse survival. Molecular analysis revealed that TF-SB controled the secretion of osteolysis-
\end{abstract}

Correspondence to: Dr Shanyu Guo, Department of General Surgery, Shanghai Ninth People's Hospital, Shanghai Jiao Tong University School of Medicine, Shanghai 200011, P.R. China

E-mail: guoshyu1@163.com

*Contributed equally

Abbreviations: TF-SB, total flavonoids from Scutellaria barbata D. Don; HPLC, high-performance liquid chromatography; PTHrP, parathyroid hormone-related protein; RANKL, receptor or activator of nuclear factor- $\mathrm{\kappa B}$ ligand; OPG, osteoprotegerin; GAPDH, glyceraldehyde 3-phosphate dehydrogenase

Key words: breast carcinoma, bone metastasis, total flavonoids from Scutellaria barbata D. Don, MDA-MB-231 cells, parathyroid hormone-related protein, osteoprotegerin, receptor activator of nuclear factor- $\kappa \mathrm{B}$ ligand related factors PTHrP and its downstream RANKL/OPG. Together, by controlling the expression of PTHrP and its downstream OPG/RANKL, TF-SB has significant inhibition effects on breast cancer bone metastasis, which indicates a new therapeutic method.

\section{Introduction}

Breast cancer is the most common malignant tumor affecting females (1). The leading cause of tumor death is metastasis, and bone is one of the most preferential metastatic sites for breast carcinoma (2). A number of studies have confirmed the theory called 'soil and seed', that the microenvironment of metastatic organs can be regarded as soil and the tumor cells are seeds. Both the soil and seeds can promote the occurrence and progress of tumor metastasis (3). Particularly, as typical soil, bone tissue possesses biological microenvironment which stores and releases various growth factors for tumor growth (4). As seeds, invasion ability of tumor cells play a decisive role (5). In fact, the main way that metastatic breast cancer cells influence bone microenvironment and achieve breast cancer bone metastasis is through regulating osteolytic destruction, which is developed by increasing the number of osteoclasts. It is abundantly clear that breast cancer cells could in turn secrete a variety of tumor derived factors, such as parathyroid hormone-related protein (PTHrP), tumor necrosis factor (TNF), vascular endothelial growth factor (VEGF) and insulin-like growth factor (IGF), to further promote osteolytic bone metastasis (6). Of these, PTHrP plays an important role. It controls the secretion of receptor activator of nuclear factor- $\kappa \mathrm{B}$ ligand (RANKL) and osteoprotegerin (OPG) to form a self-sustaining vicious circle (7-9). This vicious circle could break the balance of osteoclasts and osteoblasts, which leads to osteolytic lesion in breast cancer bone metastasis (10). Therefore, inhibiting the expression of PTHrP and its downstream RANKL/OPG is a promising approach to weaken breast cancer-induced bone metastasis and osteolysis (11).

Currently, main medical treatments for breast carcinoma bone metastasis are bisphosphonate and densumab which are inhibitors against RANKL (12-14). However, metastasis to bone is still uncontrollable and could greatly increase mortality and morbidity in breast cancer patients. For further decreasing 
skeletal morbidity and preventing disease progression, better treatments are still needed.

Scutellarin barbata D. Don is a herbal plant in Astragalus genus, known as Ban Zhi Lian in the field of traditional Chinese medicine (TCM), and has been widely used for treatment of various kinds of cancer, such as breast, colorectal, liver and lung cancer in China (15). Although the chemical constituents of Scutellarin barbata D. Don are complicated, some alkaloids, flavones, steroids have been successfully extracted and qualified. Moreover, the total flavonoids from Scutellarin barbata D. Don (TF-SB) has been proven to be the effective component and provides a favorable safety profile (16). In recent years, research in China has shown that TF-SB could suppress the growth of various tumor cells through several specific signaling pathways (17-22). In particular, it reportedly inhibits the proliferation of several human breast cancer cell lines, such as MCF-7, MCF-10A and MDA-MB-361 $(23,24)$. As a well-known TCM, Scutellarin barbata D. Don has attracted substantial research effort in exploring the molecular mechanism of its antitumor activity, but the details have not been clarified.

Based on these accurate mechanisms and existing antitumor evidence of TF-SB, our study evaluated the therapeutic effect of TF-SB on breast cancer and its bone metastasis. Furthermore, by focusing on PTHrP and its downstream RANKL/OPG, we explore the molecular mechanism of this inhibitory effect.

\section{Materials and methods}

Reagents and antibodies. Dulbecco's modified Eagle's medium (DMEM) (21063029; Invitrogen Life Technologies, Carlsbad, CA, USA), 10\% fetal bovine serum (FBS, 0500; ScienCell Research Laboratories, Carlsbad, CA, USA), trypsin-EDTA (15400054; Invitrogen Life Technologies), MTT (M2128; Sigma-Aldrich, St. Louis, MO, USA), 4\% paraformaldehyde (sc-281692; Santa Cruz Biotechnology, Inc., Santa Cruz, CA, USA), DMSO (25-950-CQC; Cellgro, Manassas, VA, USA), methanol (K977-4L; Amresco LLC, Solon, OH, USA), Transwell (3412; Corning Costar, Inc., Corning, NY, USA), Matrigel (356234; BD Biosciences, Franklin Lakes, NJ, USA), Transcriptor First Strand cDNA Synthesis kit (04897030001) and FastStart Universal SYBR-Green Master (04913914001) (both from Roche Diagnostics, Indianapolis, IN, USA), DNase I, RNase-free kit (EN0521; Thermo Fisher Scientific, Inc., Waltham, MA, USA), TRIzol (Hualan Biological Engineering Inc., Henan, China), antibodies against PTHrP (5652-100; BioVision Research Products, Mountain View, CA, USA), RANKL (GTX59855; GeneTex, Inc., Irvine, CA, USA), OPG (5312-100; BioVision Research Products) and GAPDH (622101; BioLegend, San Diego, CA, USA), HRP-conjugated antibodies against mouse IgG (BW20330016; Bioworld Technology Co., Ltd., Nanjing, China), enhanced chemiluminescence western blotting kit (32109; Pierce, Rockford, IL, USA).

Preparation of TF-SB. The whole herbs of Scutellaria barbata D. Don were purchased from a Chinese herbal medicine store called Tong Ren Tang (Shanghai, China) and verified by TCM Department of Shanghai Ninth People's Hospital (Shanghai, China). The original plant materials were prepared through drying, coarse grinding, and 95\% alcohol extraction twice. Secondly, after the evaporation and concentration, $\mathrm{pH}$ was adjusted to 2.0. The crude extracts were filtered through an AB-8 macroporous adsorption resin column. Then distilled water and $70 \%$ ethanol were used to elute TF-SB. Finally, the experimental doses of TF-SB were determined according to pharmacopoeia, references and results of pre-tests. In vitro experiments, the concentrations of TF-SB were as follows: 40, $80,120,160$ and $200 \mu \mathrm{g} / \mathrm{ml}$, but in vivo, the concentrations were 50,100 and $200 \mathrm{mg} / \mathrm{ml}$.

High-performance liquid chromatography (HPLC) analysis. TF-SB was analyzed through the HPLC system (Agilent 1100; Agilent Technologies, Inc., Santa Clara, CA, USA). According to pharmacopoeia and relevant references, purified scutellarein, apigenin, baicalein and luteolin were chosen as reference substances. Then $100 \mathrm{mg}$ TF-SB and these reference substances were respectively dissolved in $50 \mathrm{ml}$ methanol solution. Measuring wavelength was set to $335 \mathrm{~nm}$ and the sample amount was $20 \mu \mathrm{l}$. Apigenin, scutellarin, baicalein and luteolin were identified as the main component of TF-SB (Fig. 1).

Cell line and cell culture. Breast cancer cell line MDA-MB-231 was the gift from the Orthopaedic Laboratory of Shanghai Ninth People's Hospital and was maintained in Tissue Engineering Laboratory of Shanghai Ninth People's Hospital. These cells were cultured in DMEM medium, supplemented with $10 \%$ FBS and penicillin-streptomycin-amphotericin B $\left(1 \times 10^{5} \mathrm{U} / \mathrm{l}\right)$. Cells were maintained at $37^{\circ} \mathrm{C}$ and $5 \% \mathrm{CO}_{2}$ under humidified atmosphere. Through changing the culture media every two days and passaging every three days, the cell lines were kept in optimal density and state. The cells in logarithmic phase could be used in the in vitro experiment.

Animals. Thirty female athymic BALB/c nude mice (4 weeks of age) were provided by Experimental Animal Center of Shanghai Ninth People's Hospital. These mice were housed five per cage and kept in the same condition (specific pathogen-free, $26-27^{\circ} \mathrm{C}$, humidity, 12-h light/dark cycle, free access to water and food) strictly according to international ethical guidelines. Animal procedures were strictly in accordance with International Ethical Guidelines and National Institutes of Health Guide concerning the Care and Use of Laboratory Animals, and protocols were approved by the Ethics Committee of Human Experimentation of Shanghai Ninth People's Hospital. Observing the animals twice a day and starting experiments after these mice had accustomed to the new environment.

Cell viability test. Viability of TF-SB was determined by the MTT test. Cells were manipulated to cell suspension, seeded into 96-well plates at the density of $1 \times 10^{4}$ and cultured for $12 \mathrm{~h}$. An amount of $100 \mu \mathrm{l}$ TF-SB solution (0, 40, 80, 120, 160 or $200 \mu \mathrm{g} / \mathrm{ml}$ ) was added into corresponding group and incubated at $37^{\circ} \mathrm{C}$ and $5 \% \mathrm{CO}_{2}$ under humidified atmosphere for $48 \mathrm{~h}$. Then the media in these plates were removed and $20 \mu \mathrm{l}$ of MTT reagent was added into each well. After being incubated as before for $4 \mathrm{~h}, 100 \mu \mathrm{l}$ of mixture which contains SDS, isobutanol and $\mathrm{HCl}$ was added. After being shaked and incubated as before for $8 \mathrm{~h}$, the absorbance of each well was read at $540 \mathrm{~nm}$ by ELISA. Tumor inhibition rate $=($ mean 


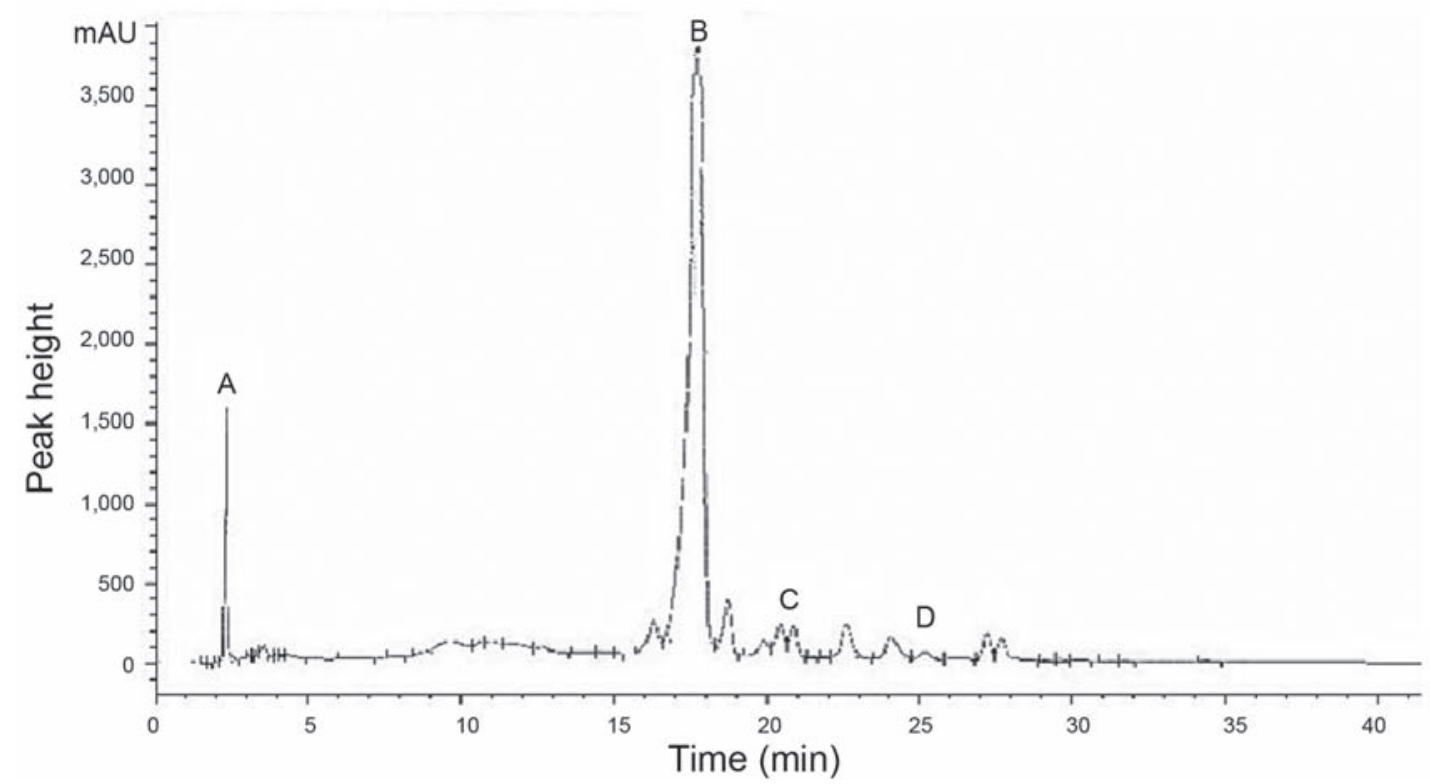

Figure 1. High-performance liquid chromatography (HPLC) analysis of TF-SB and control sample. There were four peaks in HPLC, which were identified as (A) apigenin, (B) scutellarin, (C) baicalein and (D) luteolin.

absorbance of control group - mean absorbance of TF-SB group)/(mean absorbance of control group) x100\%.

Wound healing assay. The ability of cell migration was tested by wound healing assay. MDA-MB-231 cells were seeded into 6 -well plates to a density of $5 \times 10^{5} /$ well and cultured for $12 \mathrm{~h}$. Then $200 \mu \mathrm{l}$ pipette tip was used to scratch cells in the centre points of the plates. After washing twice with phosphate-buffered saline (PBS), $100 \mu \mathrm{l}$ of TF-SB solution ( 0 , $40,80,120,160$ or $200 \mu \mathrm{g} / \mathrm{ml}$ ) was added into corresponding group and incubated at $37^{\circ} \mathrm{C}$ and $5 \% \mathrm{CO}_{2}$ under humidified atmosphere for $48 \mathrm{~h}$. The scratch areas of these groups were photographed at $0,6,12$ and $24 \mathrm{~h}$. Image J software was used to set boundaries and count the number of migrated cells in these areas. The inhibition rate of cell migration $=$ (mean number of migrated cells in control group - mean number of migrated cells in TF-SB group)/(mean number of migrated cells in control group) $\mathrm{x} 100 \%$.

Cell invasion test. Transwell chamber was used to perform the cell invasion assay. Ten microliters of fibronectin $(0.5 \mathrm{~g} / \mathrm{l})$ was used to coat the outside of $8-\mu \mathrm{m}$ pore Transwell chamber membrane and Matrigel was used inside. Fifty microliters of FBS $(10 \mathrm{~g} / \mathrm{l})$ was used to block binding sites. Then $200 \mu \mathrm{l}$ of MDA-MB-231 cell suspensions $\left(5 \times 10^{5} / \mathrm{ml}\right)$ with various concentrations $(0,40,80,120,160$ and $200 \mu \mathrm{g} / \mathrm{ml})$ of TF-SB were added into Transwell chambers. Then these Transwell chambers were set to keep suspension in 24-well plates which contain $500 \mu \mathrm{l}$ of DMEM. After being incubated at $37^{\circ} \mathrm{C}$ and $5 \% \mathrm{CO}_{2}$ under humidified atmosphere for $24 \mathrm{~h}$, residual cells in the Transwell chambers were wiped off with cotton swabs. The invading cells were fixed with methanol, stained with toluidine blue, washed with PBS, photographed under microscope and counted. The inhibition rate of cell invasion $=$ (mean number of cells in control group - mean number of cells in TF-SB group)/(mean number of cells in control group) $\mathrm{x} 100 \%$.
Breast cancer bone metastasis nude mouse model. One hundred microliters of MDA-MB-231 cell suspension $\left(8 \times 10^{7} / \mathrm{ml}\right)$ mixed with Matrigel (1:1) was injected into the femur medullary cavity of 24 nude mice to establish breast cancer bone metastasis nude mouse model. After two weeks, microcomputed tomography (micro-CT) (IVIS Quantum FX) and in vivo bioluminescence imaging (IVIS Lumina XR) (both from Caliper Life Sciences, Hopkinton, MA, USA) were used to identify neoplasm tissues and pick-out successful models. Then the nude mice were randomized into 4 groups $(n=10)$ and given a gavage of distilled water $(20 \mathrm{ml} / \mathrm{kg})$ which contained corresponding concentrations $(0,50,100$ or $200 \mathrm{mg} / \mathrm{ml})$ of TF-SB for 4 weeks. Survival rates of each group were recorded every week.

Micro-CT and in vivo bioluminescence imaging. The mice were anesthetized and placed into a dark room in a prone position. We used micro-CT to make full body scans and 3D reconstruction of mice in the animal laboratory of Huashan Hospital (Shanghai, China). Micro-CT was for observing tumor mass and bone destruction. Then in vivo bioluminescence imaging technique was performed in the same laboratory to identify the property of lumps and observing luminous intensity by setting MDA-MB-231 cell suspension as control every week. Tumor weights were calculated by measuring, recording and comparing luminous intensities between groups, which were evaluated according to the total number of photons detected by in vivo bioluminescence imaging technique. The tumor growth inhibition levels of TF-SB groups were measured by comparing tumor weights with control group.

Tumor and bone histology. Tumor tissues and corresponding bone tissues were fixed in paraformaldehyde for 3 days and decalcified in EDTA for 3 weeks. Then these tissues were trimmed into homogeneous sections, embedded in paraffin and serially cut for pathological examination. Hematoxylin 
and eosin (H\&E) staining was for contrasting histological characteristics and tartrate-resistant acid phosphatase (TRAP) staining was for observing osteoclast cells. TRAP-positive cells were photographed and quantified in same magnification, and data were expressed as number of osteoclasts per field.

Quantitative polymerase chain reaction ( $q P C R)$ of PTHrP, $O P G$ and RANKL. The mRNA expression levels were tested by qPCR (25). After extracting from tumor tissue or corresponding bone tissue with TRIzol reagent, $1 \mu 1$ of total RNA was added into nucleic acid protein detector (ND-1000; NanoDrop Technologies, Inc., Wilmington, DE, USA) to measure its concentration and $\mathrm{OD}_{260} / \mathrm{OD}_{280}$ ratio. According to the qPCR kit instruction, total RNA was reverse transcribed into cDNA which worked as template. PCR proceeded in a two-step method. PTHrP, OPG and RANKL were the objectives, and GAPDH was used as internal control. PTHrP forward, 5'-ATCGCAGAAATCCACACAGC-3' and reverse, 5'-TCATC ATCAGACCCAAATCG-3', product length was $110 \mathrm{bp}$, annealing temperature was $58^{\circ} \mathrm{C}$; OPG forward, $5^{\prime}$-CCTTGCC CTGACCACTCTTA-3' and reverse, 5'-CCCTTCCTCACACT CACACA-3', product length was $141 \mathrm{bp}$, annealing temperature was $60^{\circ} \mathrm{C}$; RANKL forward, 5'-CTGATGAAAGGAGGGAG CAC-3' and reverse, 5'-AAGGGTTGGACACCTGAATG-3', product length was $127 \mathrm{bp}$, annealing temperature was $60^{\circ} \mathrm{C}$; GAPDH forward, 5'-CAGGAGGCATTGCTGATGAT-3' and reverse, 5'-GAAGGCTGGGGCTCATTT-3', product length was $138 \mathrm{bp}$, annealing temperature was $60^{\circ} \mathrm{C}$. The expression of PTHrP, OPG, RANKL and GAPDH were determined by converting the fluorescence intensity into corresponding level of mRNA and obtaining amplification curve. Finally, the $2^{-\Delta \Delta \mathrm{Ct}}$ method was used for calculating the relative quantification of mRNA.

Western blot analysis of PTHrP, OPG and RANKL. The protein expression levels of PTHrP, OPG and RANKL were tested by western blot analysis kit. Firstly, the protein concentrations of tumor tissue or corresponding tissue were extracted by lypolysis-ultracentrifugation $\left(4^{\circ} \mathrm{C}, 12,000 \mathrm{rpm}\right.$ for $2 \mathrm{~min}$ ), quantified by manufacturing a standard curve and separated by sodium dodecyl sulfate-polyacrylamide gel electrophoresis (SDS-PAGE). Secondly, these proteins were electrophoretically transferred to polyvinylidene fluoride (PVDF) membranes. The PVDF membranes were blocked with 5\% skimmed milk for $2 \mathrm{~h}$, and respectively incubated with anti-PTHrP, anti-OPG or anti-RANKL $(1: 500)$ at $4^{\circ} \mathrm{C}$ overnight. GAPDH $(1: 1,000)$ was used as internal control to verify equivalent protein loading. Then all the PVDF membranes were incubated with HRP conjugated goat anti-mouse secondary $\operatorname{IgG}(1: 2,000)$ for $1 \mathrm{~h}$ at room temperature. Each band was revealed by enhanced chemiluminescence western blotting kit, then photographed and quantified by luminescent image analyzer (LAS-3000; Fujifilm, Tokyo, Japan).

Statistical analysis. Results are presented as mean \pm SE. Statistical analysis was performed with Student's t-test. Statistical significance was determined at $\mathrm{P} \leq 0.05$. Statistical evaluation was done by SPSS 13.0 statistical software (SPSS, Inc., Chicago, IL, USA).

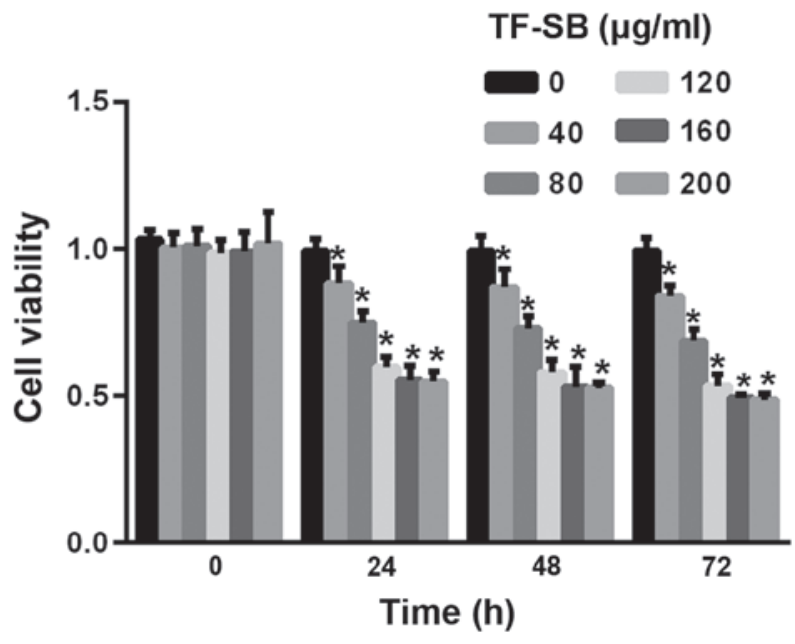

Figure 2. Proliferation inhibition rates of TF-SB on MDA-MB-231 cells and corresponding cell viability of TF-SB treated groups. Cell viability was determined by MTT assay. Cells were treated with different concentrations of TF-SB for 24,48 and $72 \mathrm{~h}$. The assay was performed in triplicate. ${ }^{*} \mathrm{P}<0.05$ compared with the control group.

\section{Results}

TF-SB inhibits MDA-MB-231 cell proliferation. TF-SB has antitumor effects in the progression of various tumors. To assess the role of TF-SB in breast cancer, we initially performed MTT assay to determine its effects on breast cancer cell viability. We chose the MDA-MB-231 cell line, which is triple-negative and high invasive, for in vitro assays. Cells were passaged into different groups and treated with corresponding doses of TF-SB for 24, 48 or $72 \mathrm{~h}$. As shown in Fig. 2, cell viability was suppressed after the TF-SB treatment with concentration from 40-200 $\mu \mathrm{g} / \mathrm{ml}$. Moreover, the anti-proliferative effects of TF-SB on MDA-MB-231 cells occured in a dose- and time-dependent manner. After $72 \mathrm{~h}$ TF-SB treatment the proliferation inhibition rates of $200 \mu \mathrm{g} / \mathrm{ml}$ group reached $51.3 \%$.

TF-SB inhibits MDA-MB-231 cell migration and invasion. Tumor cell migration and invasion are necessary parts of tumor metastasis. We performed wound healing assay and Transwell chamber assay to determine the effect of TF-SB on MDA-MB-231 cell migration and invasion. Cells were treated with various doses of TF-SB (from 40-200 $\mu \mathrm{g} / \mathrm{ml}$ ) for $24 \mathrm{~h}$. As shown in Figs. 3 and 4, cell migration and invasion in TF-SB groups were suppressed after $24 \mathrm{~h}$ treatment of TF-SB. The inhibition degrees of TF-SB on MDA-MB-231 increased in a dose-dependent manner and achieved maximum when received $200 \mu \mathrm{g} / \mathrm{ml}$ TF-SB.

TF-SB inhibits bone destruction induced by breast cancer bone metastasis while not altering tumor growth or mouse survival. Having demonstrated the effect of TF-SB on MDA-MB-231 cells, a well-established breast cancer bone metastasis model was used to evaluate its effects in vivo. To observe the degree of bone destruction, we used micro-CT and $\mathrm{H} \& \mathrm{E}$ staining to compare the imaging and histological characteristics between breast carcinoma xenograft and normal control groups (Fig. 5). Osteoclast cells were developed using TRAP staining, and 
A

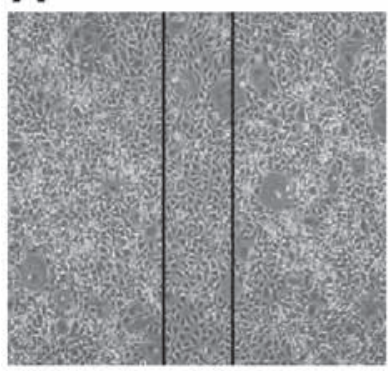

control

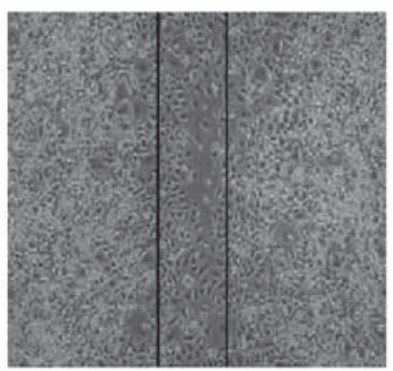

$120 \mu \mathrm{g} / \mathrm{ml}$

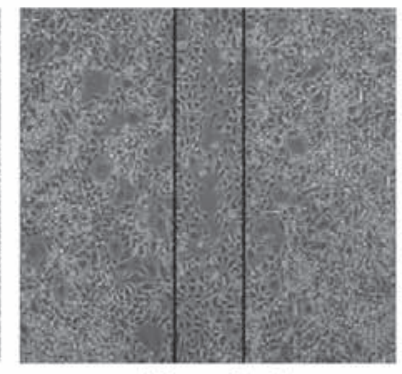

$40 \mu \mathrm{g} / \mathrm{ml}$

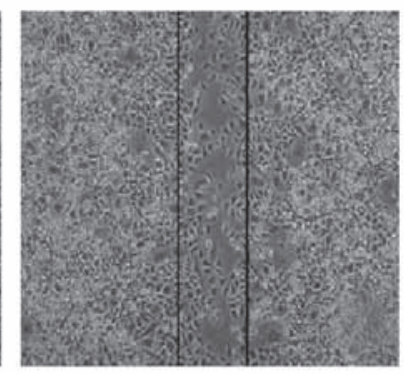

$160 \mu \mathrm{g} / \mathrm{ml}$

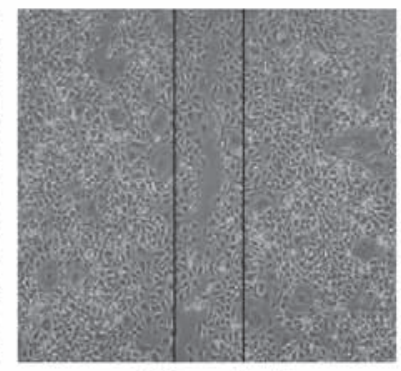

$80 \mu \mathrm{g} / \mathrm{ml}$

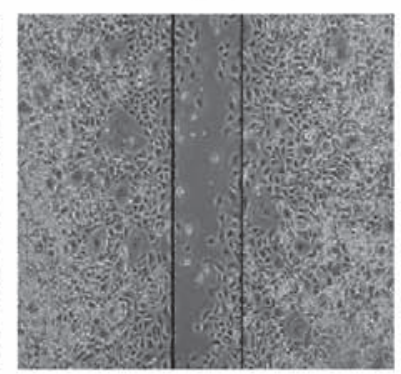

$200 \mu \mathrm{g} / \mathrm{ml}$

B

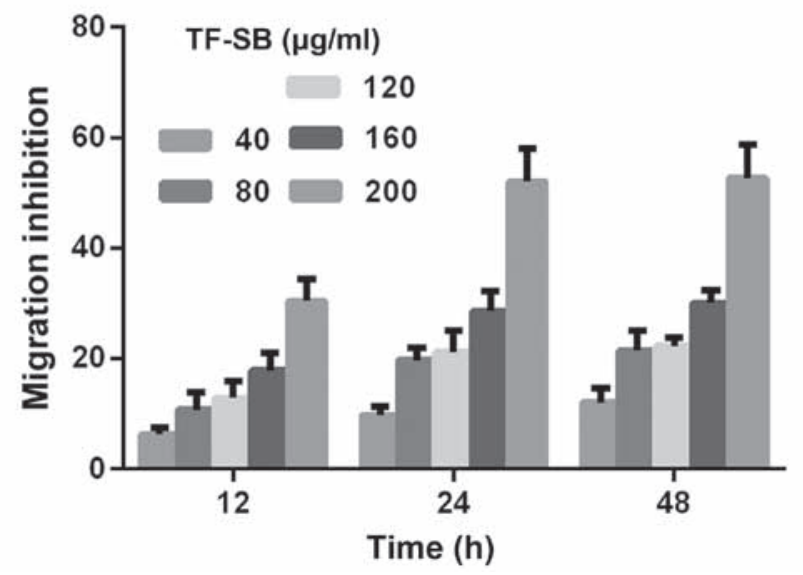

Figure 3. TF-SB inhibits migration of MDA-MB-231 cells. (A) Cell migration was determined by wound healing assay (magnification, x400). Cells were treated with different concentrations of TF-SB for $24 \mathrm{~h}$. (B) Migration inhibition rates of TF-SB on MDA-MB-231 cells. The assay was performed in triplicate.

bone destruction was measured by counting osteoclast cells. As shown in Fig. 6, the number of osteoclast cells per field in control and TF-SB treated groups (from low concentration to high) were $42 \pm 1.3,26 \pm 0.9,20 \pm 0.7,16 \pm 0.7$, respectively. Bone destruction induced by metastatic tumor cells were inhibited by TF-SB in a dose-dependent manner. But tumor weights between groups were not statistically significant until the concentration of TF-SB reached $200 \mathrm{mg} / \mathrm{ml}$ (Fig. 7). The survival in the nude mouse model was $100 \%$ until sacrifice.

Effect of TF-SB on secretion of PTHrP and its downstream RANKL/OPG in breast cancer bone metastasis. Having demonstrated the effect of TF-SB on MDA-MB-231 cells and osteoclast cells, we continued to study the underlying molecular mechanism. PTHrP and its downstream RANKL/OPG play crucial roles in tumor progression and osteolytic lesion induced by breast carcinoma bone metastasis. In order to confirm that
PTHrP pathway was responsible for explaining the inhibition effect of TF-SB, mRNA expression levels of PTHrP, RANKL and OPG were measured by qPCR assays. As shown in Fig. 8, compared with control group, PTHrP and RANKL expression levels were steeply decreased in the presence of TF-SB, but OPG expression levels were slightly increased. We concluded that the mRNA expression of PTHrP and its downstream RANKL/ OPG could be affected by TF-SB in a dose-dependent manner. Based on the positive results of qPCR, we further detected the effects of TF-SB on protein expression levels. In this study, protein expression of PTHrP, RANKL and OPG were analyzed using western blot analysis. As shown in Fig. 9, the highest expression levels of PTHrP and RANKL appeared in control group but decreased in TF-SB treated groups. In contrast, OPG expression levels had the opposite trend. The results indicated that TF-SB decreased the protein expression of PTHrP and RANKL, but promoted OPG. 
A

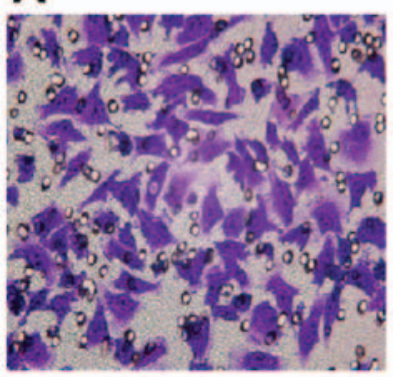

control

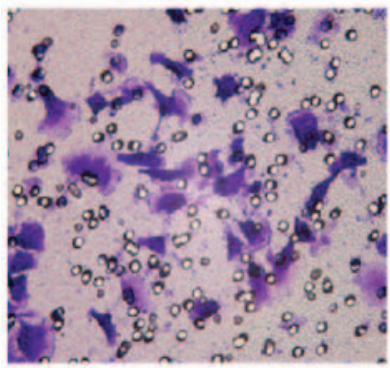

$120 \mu \mathrm{g} / \mathrm{ml}$

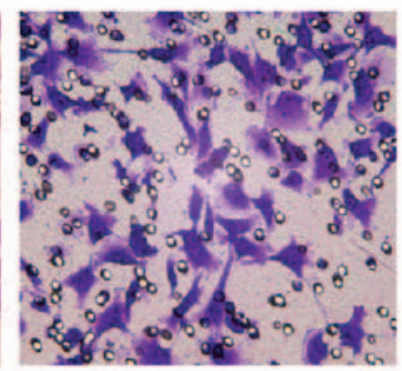

$40 \mu \mathrm{g} / \mathrm{ml}$

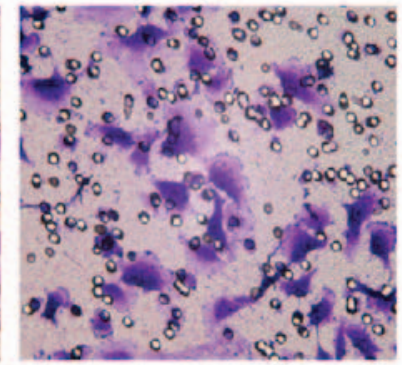

$160 \mu \mathrm{g} / \mathrm{ml}$

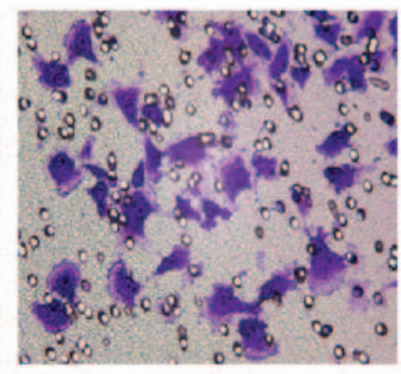

$80 \mu \mathrm{g} / \mathrm{ml}$

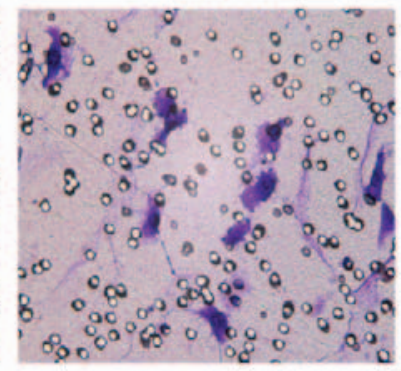

$200 \mu \mathrm{g} / \mathrm{ml}$

B

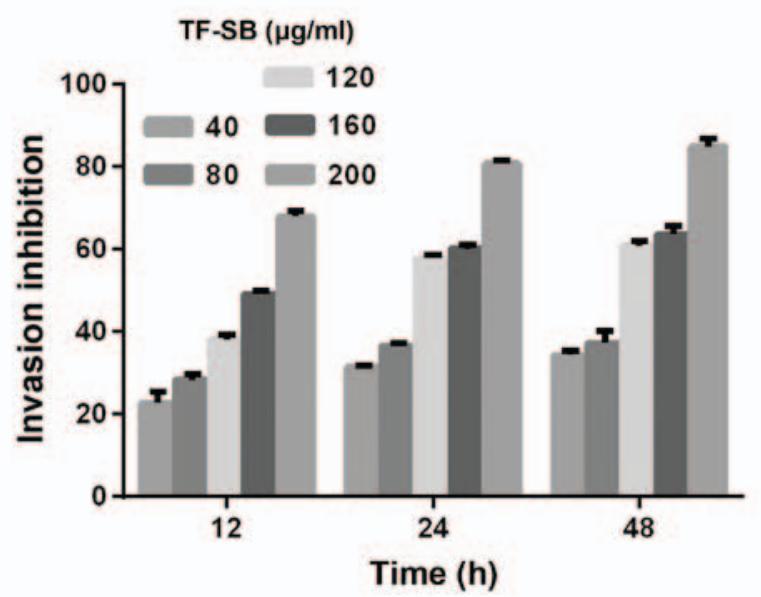

Figure 4. TF-SB inhibits invasiveness of MDA-MB-231 cells. (A) Cell invasiveness was determined by wound healing assay (magnification, $\mathrm{x} 400$ ). Cells were treated with different concentrations of TF-SB for $24 \mathrm{~h}$. (B) Invasion inhibition rates of TF-SB on MDA-MB-231 cells. The assay was performed in triplicate.

\section{Disscussion}

In the field of TCM, Scutellaria barbata D. Don has been widely used in the treatment of inflammation and tumors by clearing heat, activating blood and dissolving stasis (15). Chromatographic analysis has demonstrated that scutellarein, apigenin, baicalein and luteolin are main components to the alcohol extract of Scutellaria barbata D. Don (16). Moreover, TF-SB turned out to be the main effective component in previous studies which had favorable safety profile (14). In our study, we had confirmed the existence of these main elements in TF-SB. In recent years, Scutellarin barbata D. Don has been involved in numerous antineoplastic studies (17-22). Specific to breast cancer, alcohol extract of Scutellaria barbata D. Don has shown inhibition effects on several cell lines $(23,24)$.
It is generally known that breast carcinoma frequently spreads to bone (26). In fact, approximately $70 \%$ of patients with advanced breast cancer metastasize to the skeleton and has risk of skeletal-related events (3). Bone-target metastasis of breast cancer contains multiple biological steps (27). The interaction between bone microenvironment and metastasized breast cancer cells plays a major role (4). It has been well accepted that osteoclasts in the bone microenvironment are responsible for metastatic osteolytic lesions in bone metabolism (28). In fact, bone resorption begins with the recruitment of large activated multinuclear osteoclasts differentiated from non-functional multinuclear pre-osteoclasts (29). Breast cancer cells secrete various osteolytic factors, including PTHrP, a protein, initially identified to be the factor in charge of malignant hypercalcemia (30). In primary breast cancer, PTHrP is expressed in $50 \%$ of patients (31). But in breast cancer bone metastasis samples, 

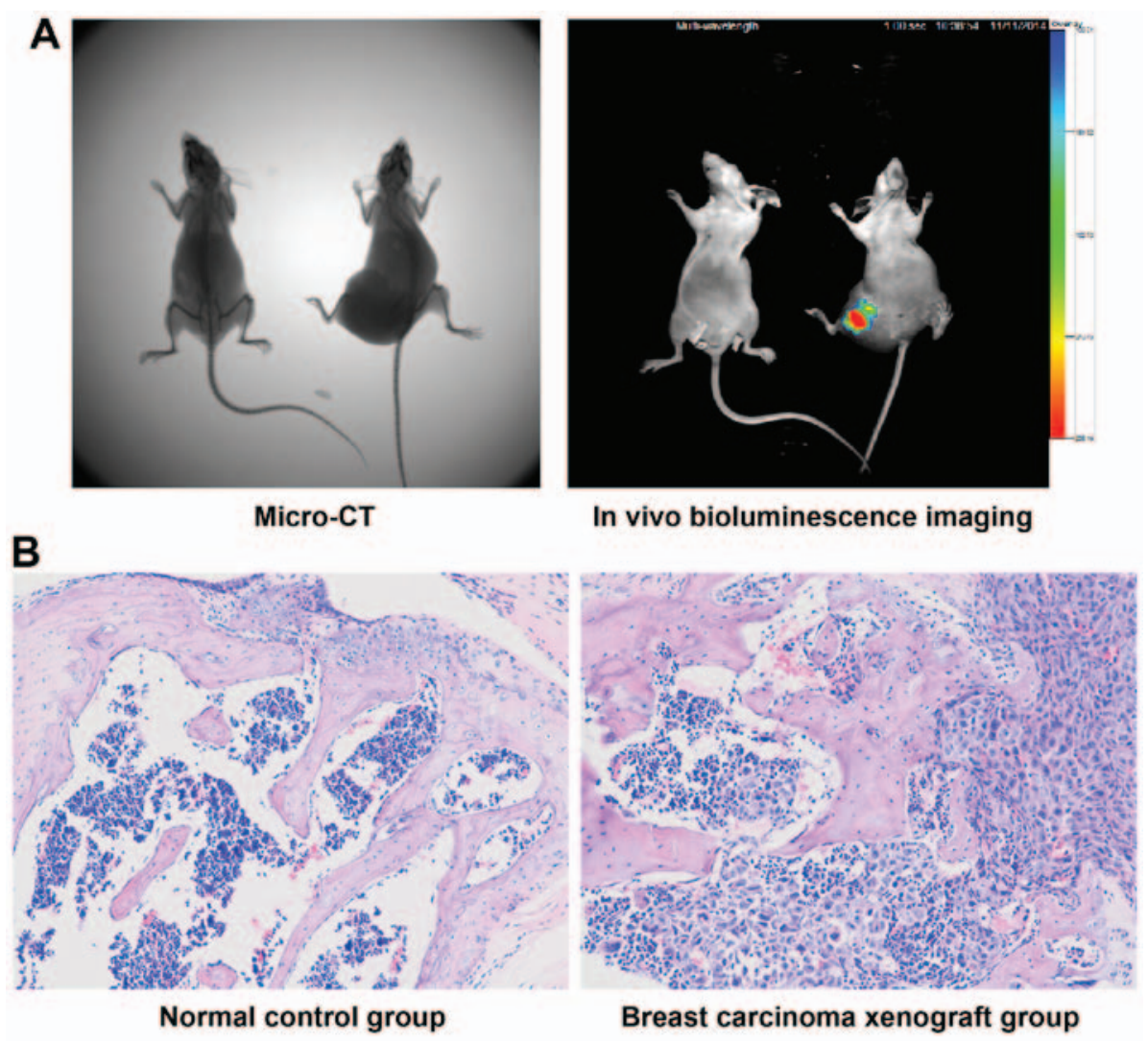

Figure 5. Identification of breast cancer bone metastasis in nude mouse model. (A) Models were measured by micro-CT and in vivo bioluminescence imaging. (B) Hematoxylin and eosin (H\&E) staining of bone tissue in normal control and breast carcinoma xenograft groups (magnification, x100).

the percent rises to $90 \%$. It is clear that tumor-derived PTHrP works as a central contributor in promoting osteolytic process of breast carcinoma bone metastasis $(7,32)$. Specifically, rather than stimulating osteoclast differentiation directly, tumor-derived PTHrP could activate stromal cells and osteoblasts to upregulate RANKL expression and cocurrently lower OPG expression (8). This forms a feed-forward pattern as mentioned above as a vicious circle with multi-interactions between malignant cells, osteoblasts, osteoclasts and bone microenvironment. RANKL is expressed on the surface of osteoblast and could interact with the receptor RANK, which is expressed on the surface of osteoclast precursor, to further activate the osteoclast differentiation (9). RANKL binding to the RANK plays a central role in the establishment of this vicious circle. This circle could finally improve tumor cell proliferation, trigger osteoclast activation and leads to osteolytic lesion of breast cancer bone metastasis. Otherwise, OPG is a decoy receptor which prevents RANKL binding to its receptor and inhibits osteoclast activation (10). So the balance between RANKL and OPG decides the degree of osteoclastic activity and bone resorption. In our study, by particularly focusing on the role of PTHrP and its downstream RANKL/ OPG, effects and relative mechanisms of TF-SB on breast cancer bone metastasis were verified at mRNA and protein levels.

As for human breast cancer cell line MDA-MB-231, owing to its high potential of invasion and metastasis, it has been identified as the ideal and most common cell line for modeling bone metastasis of breast cancer. In vitro, our laboratory firstly proved the inhibition capacity of TF-SB on cell proliferation, migration and invasion which were detected, respectively, by MTT test, wound healing assay and Transwell chamber assay.
In fact, at the experimental concentrations of TF-SB ranging from 40 to $200 \mu \mathrm{g} / \mathrm{ml}$, their inhibition tendencies were dosedependent. Evidence was shown that there was no benefit for further improving the experimental concentration. These positive results on MDA-MB-231 cell line were the basis for further study on breast cancer bone metastasis in murine model.

In vivo, we injected MDA-MB-231 cell suspension into the right femur medullary cavity of female nude mice to build breast cancer bone metastasis animal model. According to the result of random assignment, we further fed these animals with corresponding concentrations of TF-SB for one month. Then macroscopic and microcosmic angles, in vivo bioluminescence and $H \& E$ staining were used to verify nude mouse models, micro-CT and TRAP staining for observing osteolytic lesion caused by tumor cells. As the results suggested, there was no effect of TF-SB on tumor growth and mouse survival.

It had been well accepted that osteoclasts were the major cause for metastatic osteolytic lesions in bone metabolism (28). So focusing on the outcome of TRAP staining, we drew the conclusion that TF-SB could diminish osteoclast cells and ease bone destruction caused by metastatic tumor cells in bone tissue. As tumor-derived PTHrP control the secretion of RANKL and OPG, and the ratio of RANKL to OPG decides the degree of bone degradation, so inhibiting the expression of PTHrP and its downstream RANKL/OPG has been regarded as a hopeful way to impair bone destruction (7). In order to confirm the relationship between PTHrP pathway and inhibitory effects of TF-SB on breast cancer bone metastasis, we offer evidence on mRNA and protein levels. Our results suggested that TF-SB could decrease mRNA 


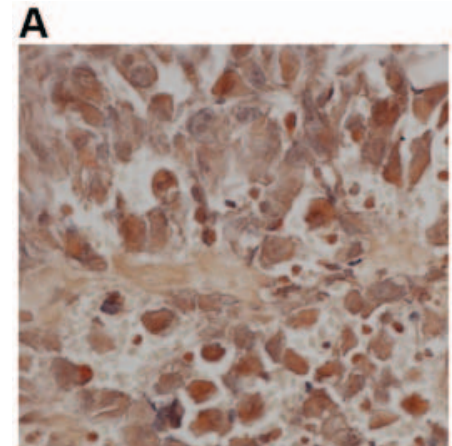

control

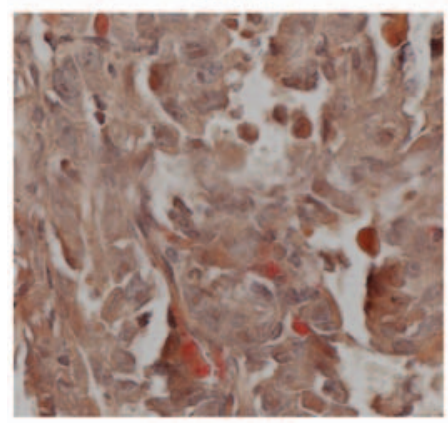

$100 \mathrm{mg} / \mathrm{ml}$

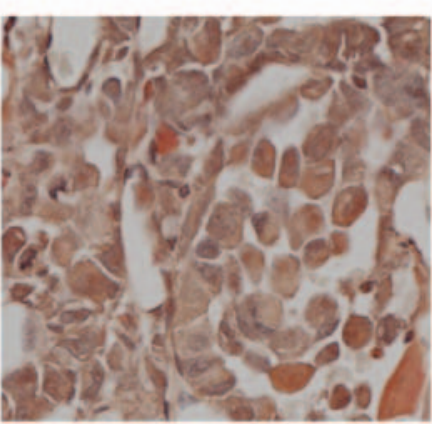

$50 \mathrm{mg} / \mathrm{ml}$

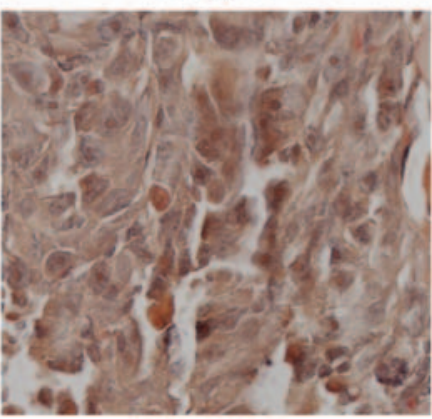

$200 \mathrm{mg} / \mathrm{ml}$

B

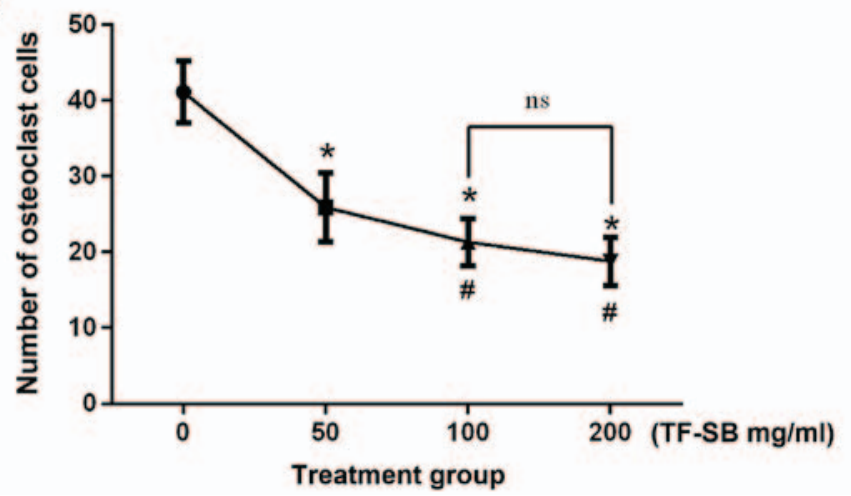

Figure 6. TF-SB inhibits bone destruction. (A) Tartrate-resistant acid phosphatase (TRAP) staining of osteoclast cells in control and TF-SB treated groups (magnification, $\mathrm{x} 400$ ). (B) The number of osteoclast cells per field in control and TF-SB treated groups. " $\mathrm{P}<0.05$ compared with the control group.

A

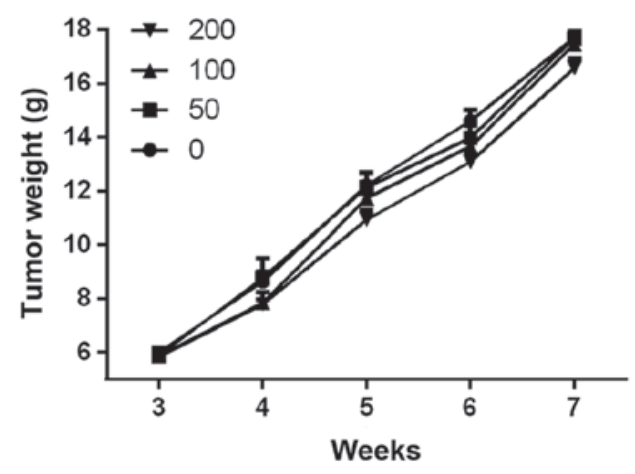

B

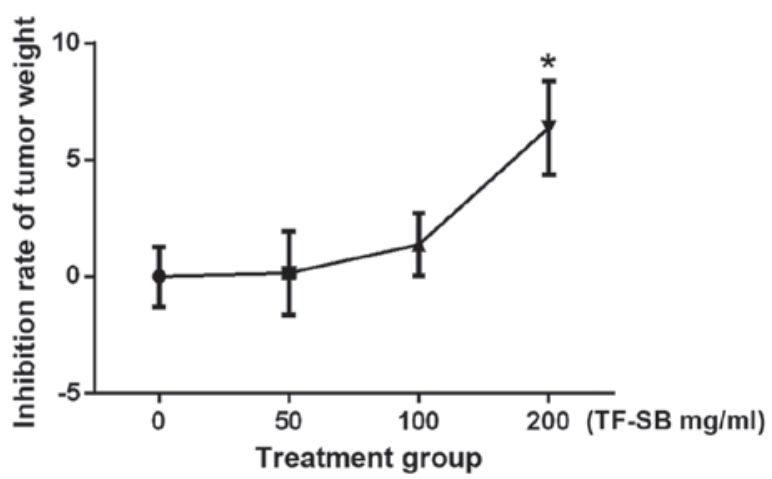

Figure 7. (A) Tumor weights and (B) corresponding inhibition rates in control and TF-SB treated groups. "P<0.05 compared with the control group.

and protein expression of PTHrP and RANKL, but on the contrary, promote OPG expression. So a positive correlation had been established between the phenomenon and mecha- nism. The data in vitro and in vivo from our study suggest that TF-SB treatment has beneficial effects on breast cancer bone metastasis by controlling the expression of PTHrP and 

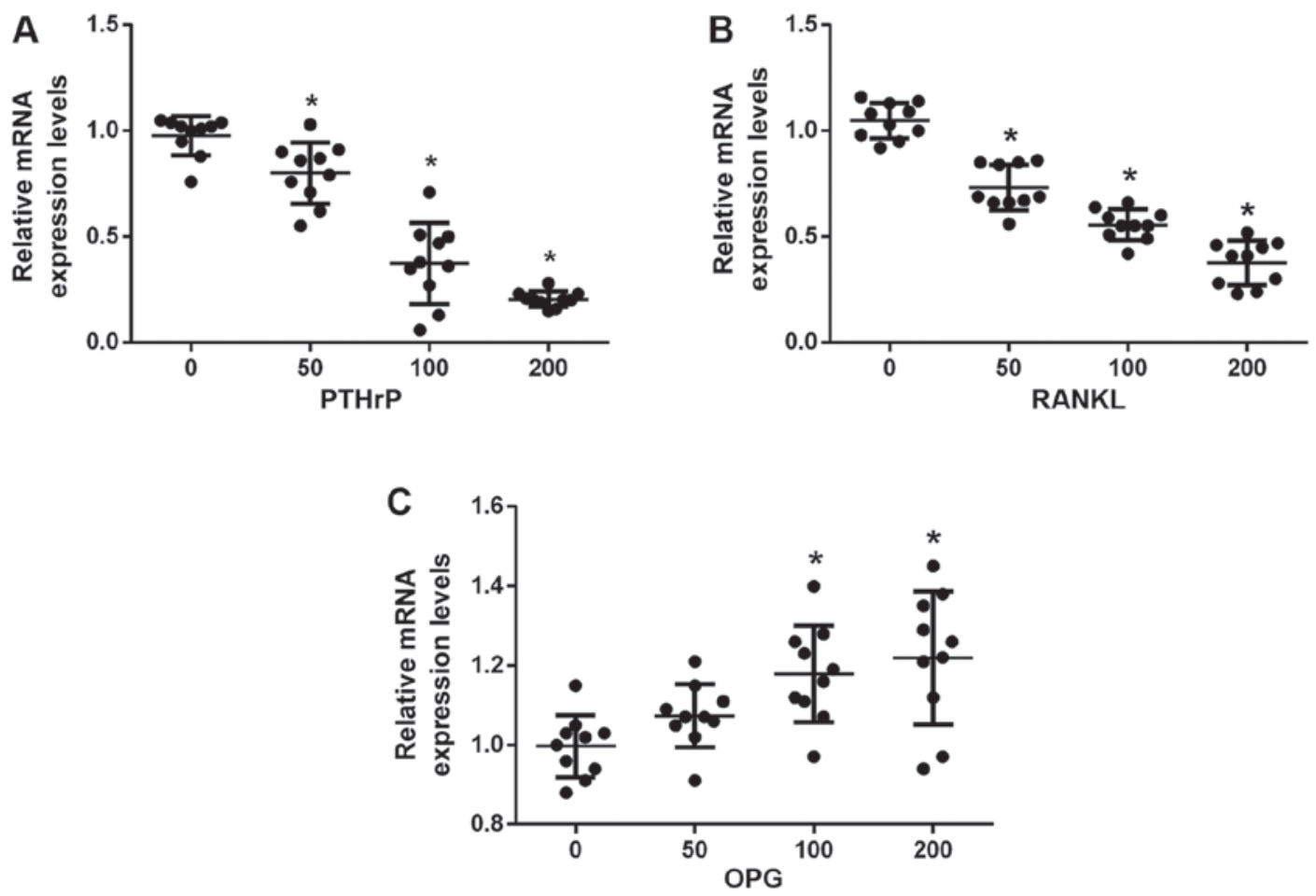

Figure 8. The mRNA expression levels of (A) parathyroid hormone-related protein (PTHrP), (B) receptor activator of nuclear factor- $\mathrm{kB}$ ligand (RANKL) and (C) osteoprotegerin (OPG) in each group. GAPDH was used as the internal control.

A

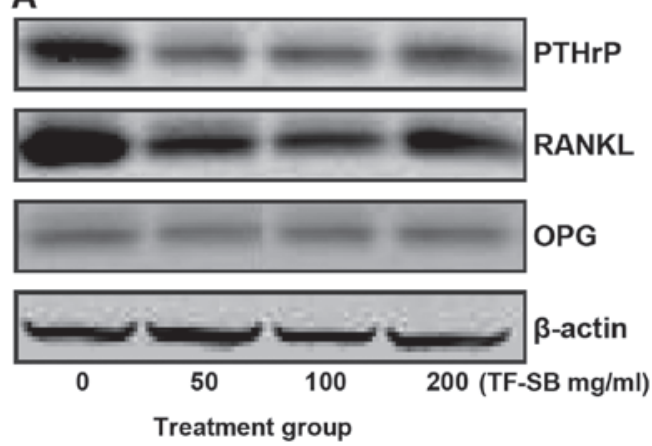

B

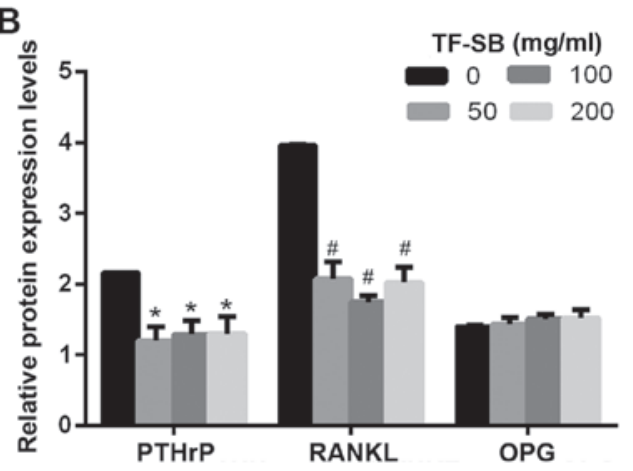

Figure 9. (A) Western blot analysis of parathyroid hormone-related protein (PTHrP), receptor activator of nuclear factor- $\kappa \mathrm{B}$ ligand (RANKL) and osteoprotegerin (OPG). $\beta$-actin was used as the internal control. (B) The protein expression levels of PTHrP, RANKL and OPG in each group. ${ }^{* \#} \mathrm{P}<0.05$ compared with the control group.

its downstream OPG/RANKL. Here we reported that TF-SB targeting the PTHrP pathway offered a new approach for therapeutic intervention in breast carcinoma bone metastasis. However, for the pursuit of more effective and selective treatments, further research could establish positive control drugs, focus on purified components of TF-SB and extend upstream of PTHrP pathway, such as TGF- $\beta$. More study should be done to resolve the unconformity between bioactivity of tumor cells and bioavailability of TF-SB.

\section{Acknowledgements}

We thank the Chemistry Experiment Center of Shanghai Normal University, the Orthopedic Laboratory and Tissue Engineering Laboratory of Shanghai Ninth People's Hospital for their assistance.

\section{Funding}

This study was supported by the Research Innovation Program of Shanghai Municiple Education Commission (grant no. 09yz79).

\section{Availability of data and material}

The data and materials generated or analysed during this study are available from the corresponding author on reasonable request.

\section{Authors' contributions}

$\mathrm{XZ}$ performed the animal experiment. WK performed the cell experiment. They made substantial contributions to design this study and were major contributors in writing the manuscript. HL analyzed and interpreted the data. As director of this study, 
SG contributed to the conception of this study, gave guidance and final approval of the version to be published. All authors read and approved the final manuscript.

\section{Ethics approval and consent to participate}

The experimental protocol was established according to the ethical guidelines and was approved by the Ethics Committee of Shanghai Ninth People's Hospital.

\section{Consent for publication}

Not applicable.

\section{Competing interests}

The authors declare that they have no competing interests.

\section{References}

1. TA S: Genetic factors in the pathogenesis of breast cancer: their role and relative importance. Nutr 127 (Suppl): 929-932, 1997.

2. Roodman GD: Mechanisms of bone metastasis. N Engl J Med 350: 1655-1664, 2004.

3. Paget S: The distribution of secondary growths in cancer of the breast. Lancet 1: 571-573, 1889.

4. Schulman KL and Kohles J: Economic burden of metastatic bone disease in the U.S. Cancer 109: 2334-2342, 2007.

5. Mundy GR: Metastasis to bone: causes, consequences and therapeutic opportunities. Nat Rev Cancer 2: 584-593, 2002.

6. Kominsky SL and Davidson NE: A 'bone' fide predictor of metastasis? Predicting breast cancer metastasis to bone. J Clin Oncol 24: 2227-2229, 2006.

7. Dougall WC: Molecular pathways: osteoclast-dependent and osteoclast-independent roles of the RANKL/RANK/OPG pathway in tumorigenesis and metastasis. Clin Cancer Res 18: 326-335, 2012.

8. Schramek D, Sigl V and Penninger JM: RANKL and RANK in sex hormone-induced breast cancer and breast cancer metastasis. Trends Endocrinol Metab 22: 188-194, 2011.

9. Kennecke H, Yerushalmi R, Woods R, Cheang MC, Voduc D, Speers CH, Nielsen TO and Gelmon K: Metastatic behavior of breast cancer subtypes. J Clin Oncol 28: 3271-3277, 2010.

10. Chiechi A, Waning DL, Stayrook KR, Buijs JT, Guise TA and Mohammad KS: Role of TGF- $\beta$ in breast cancer bone metastases. Adv Biosci Biotechnol 4: 15-30, 2013.

11. Datta NS and Abou-Samra AB: PTH and PTHrP signaling in osteoblasts. Cell Signal 21: 1245-1254, 2009.

12. Roelofs AJ, Coxon FP, Ebetino FH, Lundy MW, Henneman ZJ, Nancollas GH, Sun S, Blazewska KM, Bala JL, Kashemirov BA, et al: Fluorescent risedronate analogues reveal bisphosphonate uptake by bone marrow monocytes and localization around osteocytes in vivo. J Bone Miner Res 25: 606-616, 2010

13. Syddall SP, Ottewell PD and Holen I: Combined therapies of bone disease with bisphosphonates. Curr Pharm Des 16: 2988-2997, 2010

14. Lacey DL, Boyle WJ, Simonet WS, Kostenuik PJ, Dougall WC, Sullivan JK, San Martin J and Dansey R: Bench to bedside: elucidation of the OPG-RANK-RANKL pathway and the development of denosumab. Nat Rev Drug Discov 11: 401-419, 2012.

15. Chinese Pharmacopoeia Commission: Pharmacopoeia of the People's Republic of China. China Medical Science and Technology Press, Beijing, pp109-110, 2010.
16. Ruan C, Xiao XH and Li GK: Microwave-assisted extraction coupled with countercurrent chromatography for the rapid preparation of flavonoids from Scutellaria barbata D. Don. J Sep Sci 37: 1364-1369, 2014

17. Lin J, Chen Y, Cai Q, Wei L, Zhan Y, Shen A, Sferra TJ and Peng J: Scutellaria barbata D. Don inhibits colorectal cancer growth via suppression of multiple signaling pathways. Integr Cancer Ther 13: 240-248, 2014.

18. Wei L, Lin J, Wu G, Xu W, Li H, Hong Z and Peng J: Scutellaria barbata $\mathrm{D}$. Don induces G1/S arrest via modulation of p53 and Akt pathways in human colon carcinoma cells. Oncol Rep 29: 1623-1628, 2013.

19. Wei L, Lin J, Xu W, Cai Q, Shen A, Hong Z and Peng J: Scutellaria barbata $\mathrm{D}$. Don inhibits tumor angiogenesis via suppression of Hedgehog pathway in a mouse model of colorectal cancer. Int J Mol Sci 13: 9419-9430, 2012.

20. Gao J, Lu WF, Dai ZJ, Lin S, Zhao Y, Li S, Zhao NN, Wang XJ, Kang HF, Ma XB, et al: Induction of apoptosis by total flavonoids from Scutellaria barbata D. Don in human hepatocarcinoma MHCC 97-H cells via the mitochondrial pathway. Tumour Biol 35: 2549-2559, 2014.

21. Tang PM, Chan JY, Zhang DM, Au SW, Fong WP, Kong SK, Tsui SK, Waye MM, Mak TC and Fung KP: Pheophorbide a, an active component in Scutellaria barbata, reverses P-glycoprotein-mediated multidrug resistance on a human hepatoma cell line R-HepG2. Cancer Biol Ther 6: 504-509, 2007.

22. Yin X, Zhou J, Jie C, Xing D and Zhang Y: Anticancer activity and mechanism of Scutellaria barbata extract on human lung cancer cell line A549. Life Sci 75: 2233-2244, 2004.

23. Shi Y, Zhou ZY, Cheng LJ and Lin MB: The effects of scutellarin in barbated skullcup herb on galactophore cancer MCF-7 cell proliferation and invasion potentia. Acta Academiae Medicinae Jiangxi 49: 12-14, 2009 (In Chinese).

24. Fong S, Shoemaker M, Cadaoas J, Lo A, Liao W, Tagliaferri M, Cohen I and Shtivelman E: Molecular mechanisms underlying selective cytotoxic activity of BZL101, an extract of Scutellaria barbata, towards breast cancer cells. Cancer Biol Ther 7: 577-586, 2008.

25. Bustin SA and Mueller R: Real-time reverse transcription PCR (qRT-PCR) and its potential use in clinical diagnosis. Clin Sci (Lond) 109: 365-379, 2005.

26. Weilbaecher KN, Guise TA and McCauley LK: Cancer to bone: a fatal attraction. Nat Rev Cancer 11: 411-425, 2011.

27. Kearns AE, Khosla S and Kostenuik PJ: Receptor activator of nuclear factor kappaB ligand and osteoprotegerin regulation of bone remodeling in health and disease. Endocr Rev 29: 155-192, 2008

28. Blanco MA and Kang Y: Signaling pathways in breast cancer metastasis - novel insights from functional genomics. Breast Cancer Res 13: 206, 2011

29. Boyle WJ, Simonet WS and Lacey DL: Osteoclast differentiation and activation. Nature 423: 337-342, 2003.

30. Yin JJ, Selander K, Chirgwin JM, Dallas M, Grubbs BG, Wieser R, Massagué J, Mundy GR and Guise TA: TGF-beta signaling blockade inhibits PTHrP secretion by breast cancer cells and bone metastases development. J Clin Invest 103: 197-206, 1999.

31. Hastings RH, Burton DW, Nefzi A, Montgrain PR, Quintana R and Deftos LJ: Combinatorial library discovery of small molecule inhibitors of lung cancer proliferation and parathyroid hormone-related protein expression. Cancer Biol Ther 10: 1067-1075, 2010

32. Soki FN, Park SI and McCauley LK: The multifaceted actions of PTHrP in skeletal metastasis. Future Oncol 8: 803-817, 2012.

This work is licensed under a Creative Commons Attribution-NonCommercial-NoDerivatives 4.0 International (CC BY-NC-ND 4.0) License. 